\title{
Selenoprotein $\mathrm{K}$ and Protein Palmitoylation
}

\author{
Gregory J. Fredericks and Peter R. Hoffmann
}

\begin{abstract}
Significance: Selenoprotein K (SelK) is an endoplasmic reticulum (ER) membrane protein, and its expression is sensitive to dietary selenium levels. A recently described role for SelK as a cofactor in catalyzing protein palmitoylation reactions provides an important link between low dietary selenium intake and suboptimal cellular functions that depend on this selenoprotein for palmitoylation. Recent Advances: A recent breakthrough provided insight into the contribution of SelK to calcium $\left(\mathrm{Ca}^{2+}\right)$ flux in immune cells. In particular, SelK is required for palmitoylation of the $\mathrm{Ca}^{2+}$ channel protein, inositol-1,4,5-triphosphate receptor (IP3R) in the ER membrane. Without this post-translational modification, expression and function of the IP3R is impaired. SelK is required for palmitoylation of another transmembrane protein, CD36, and very likely other proteins. SelK serves as a cofactor during protein palmitoylation by binding to the protein acyltransferase, DHHC6, thereby facilitating addition of the palmitate via a thioester bond to the sulfhydryl group of cysteine residues of target proteins. Critical Issues: The association of DHHC6 and SelK is clearly important for immune cell functions and possibly other cell types. The step in the DHHC6 catalyzed S-acylation reaction on which SelK acts remains unclear and possible mechanisms of how the kinetics of the reaction are impacted by SelK binding to DHHC6 are presented here. Future Directions: Uncovering the specific role of SelK in promoting DHHC6 catalyzed protein palmitoylation may open a new line of inquiry into other selenoproteins playing similar roles as cofactors for different enzymatic processes. Antioxid. Redox Signal. 23, 854-862.
\end{abstract}

\section{Introduction}

$\mathbf{T}$ HE HUMAN SELENOPROTEOME comprises a diverse group of proteins that play important roles in many biological processes throughout the body. The one feature common to all selenoproteins is the presence within the peptide sequence of the 21st amino acid, selenocysteine (Sec). Given the reducing capacity of Sec, it is not surprising that many selenoproteins exhibit oxidoreductase enzymatic activity and function primarily as antioxidants or redox-regulating enzymes $(26,33)$. However, it has become increasingly clear that some members of the selenoprotein family may play important roles other than directly catalyzing oxidoreductase reactions.

An early example of alternative biological roles was the exciting finding by Ursini et al. that phospholipid hydroperoxide glutathione peroxidase (PHGPx; GPx4) functions both as an antioxidant enzyme and as a nonenzymatic structural protein within the midpiece of mature spermatozoa (41). This prompted many researchers to think about alternative roles for the Sec within some selenoproteins, particularly those lacking the $\mathrm{C}-\mathrm{X}-\mathrm{X}-\mathrm{U}$ catalytic domains found in established selenoenzymes. It has also become clear that certain selenoproteins may have multiple functional roles within cells, and that these different roles likely depend on the partner proteins or complexes with which the selenoproteins interact. This was vividly demonstrated in a recent study by Turanov et al., in which selenoprotein S (SelS) was shown by mass spectrophotometry to interact with nearly 200 protein partners (40). By binding to different partners, SelS may contribute to the maintenance of endoplasmic reticulum (ER) homeostasis, ER-associated protein degradation (ERAD) and inflammation, or the maintenance and intracellular membrane-based transport of protein complexes.

Another ER transmembrane selenoprotein that appears to have multiple biological roles is selenoprotein $\mathrm{K}(\mathrm{SelK})$, and its function may depend on its interactions with different proteins. SelK can bind to ERAD components, including Derlin-1 and the p97 ATPase, and is involved in transport of

Department of Cell and Molecular Biology, John A. Burns School of Medicine, University of Hawaii, Honolulu, Hawaii. 
glycosylated misfolded proteins and regulation of ER stress in some cell lines $(9,38)$. Overexpression of SelK in cardiomyocytes has been implicated in potential antioxidant responses (23), and purified human SelK was shown to reduce phospholipid hydroperoxides (22). This may suggest a role for SelK in ER membrane repair, although it remains to be determined whether this occurs in vivo.

Our laboratory found that SelK was expressed at relatively high levels in immune cells, and our investigations into the in vivo role of SelK in the immune system were initiated through the generation of $\mathrm{SelK}^{-/-}$mice (43). Immune cells from SelK ${ }^{-l-}$ mice did not show indications of ER stress in the resting or activated state, perhaps due to overlapping roles of SelK and SelS related to the regulation of ER stress. We instead found that receptor-mediated calcium $\left(\mathrm{Ca}^{2+}\right)$ flux was impaired in various immune cells, including $\mathrm{SelK}^{-/-} \mathrm{T}$ cells, neutrophils, and macrophages. This impaired $\mathrm{Ca}^{2+}$ flux led to defects in a variety of immune cell functions, including migration, proliferation, and oxidative burst that were associated with higher susceptibility to West Nile virus infection due to impaired viral clearance (43).

SelK-dependent $\mathrm{Ca}^{2+}$ flux is regulated not only by bioavailable selenium but also by proteolytic modulation through the calpain/calpastatin enzyme system (17). Interestingly, calpain regulation of SelK was only found in innate immune cells such as macrophages, but not in T or B cells. The $\mathrm{Ca}^{2+}$ flux mediated by SelK was found to be crucial for ERK activation and nitric oxide production required for phagocytosis of IgG-opsonized particles by macrophages (16). In addition to IgG-opsonized particles, oxidized lowdensity lipoprotein (LDL) uptake was impaired in SelK ${ }^{-/-}$ macrophages, and this led to reduced foam cell formation and atherosclerosis (25). The latter study provided a major breakthrough in understanding SelK's role in regulating immune cell functions due to our discovery that SelK deficiency led to reduced palmitoylation of the receptor for oxidized LDL, CD36.

Palmitoylation is a post-translational modification involving the reversible addition of the 16-carbon fatty acid, palmitate, to cysteine residues through a thioester bond (3), and this modification can facilitate membrane association of cytosolic proteins or can promote stable expression of transmembrane proteins $(7,37)$. The requirement of SelK for palmitoylation of CD36 and the impaired $\mathrm{Ca}^{2+}$ flux in SelKdeficient immune cells led us to form a new hypothesis: The $\mathrm{Ca}^{2+}$ channel protein in the ER membrane that facilitates $\mathrm{Ca}^{2+}$ flux in stimulated immune cells, the inositol-1,4,5-triphosphate receptor (IP3R), is palmitoylated in an SelK-dependent manner and this is required for its stable expression. This was indeed found to be the case as demonstrated in our recent study showing the role of SelK in IP3R palmitoylation (13). These findings have opened up an entirely new line of investigation into the biological role of SelK in protein palmitoylation and suggest the possibility of this and other selenoproteins serving as cofactors for enzymatic reactions instead of directly catalyzing reactions as selenoenzymes.

\section{Structure and Localization of SelK}

SelK was initially described as a "G-rich protein" in Drosophila (29), and the topology of G-rich protein, also called Drosophila SelK (dSelK), was described as a single-pass transmembrane protein in the Golgi with the Sec residue near the C-terminus in the cytosol. Mammalian SelK was identified in the 2003 hallmark paper by Kryukov et al. that characterized the 25 human selenoproteins (19). Similar to dSelK, the mammalian SelK is a small transmembrane protein comprising 94-amino-acid proteins with the Sec residue near the $\mathrm{C}$ terminus (Fig. 1). SelK amino-acid sequences from human and mouse share $91 \%$ identity. The localization of SelK to the ER membrane was verified in several independent studies $(23,38$, 43). Interestingly, early reports suggested plasma membrane localization of SelK by immunostaining and electron microscopy (19). However, this may have been due to the fact that regions of ER, which are commonly referred to as puncta, come in close proximity (within $10-25 \mathrm{~nm}$ ) to the plasma membrane (44). To date, no canonical ER localization signal or mechanism for exclusive ER localization has been established and some SelK protein residing in the plasma membrane cannot be ruled out.

The region of SelK located in the ER lumen has no identifiable features. The cytosolic region of SelK is rich in both prolines and glycines, with a calpain-2 cleavage site present in the cytosolic region between amino-acid residues Arg81 and Gly 82 (17). Purified human SelK was found to dimerize through the formation of intermolecular diselenide bonds (22), but it remains unclear whether this occurs in the ER membrane of mammalian cells. Other than the Sec residue, the most distinctive feature of SelK is a predicted Src homology 3 (SH3) binding domain. Canonical SH3-binding sequences (R/K-X-X-P-X-X-P) promote interactions with $\mathrm{SH} 3$ domains on other proteins (46). These $\mathrm{SH} 3 / \mathrm{SH} 3$ binding domain interactions enable functional complexes to form between partner proteins (18).

Interestingly, the location of the calpain cleavage site between the SH3 domain and the Sec residue suggests that the cleaved version of SelK may be able to retain its binding

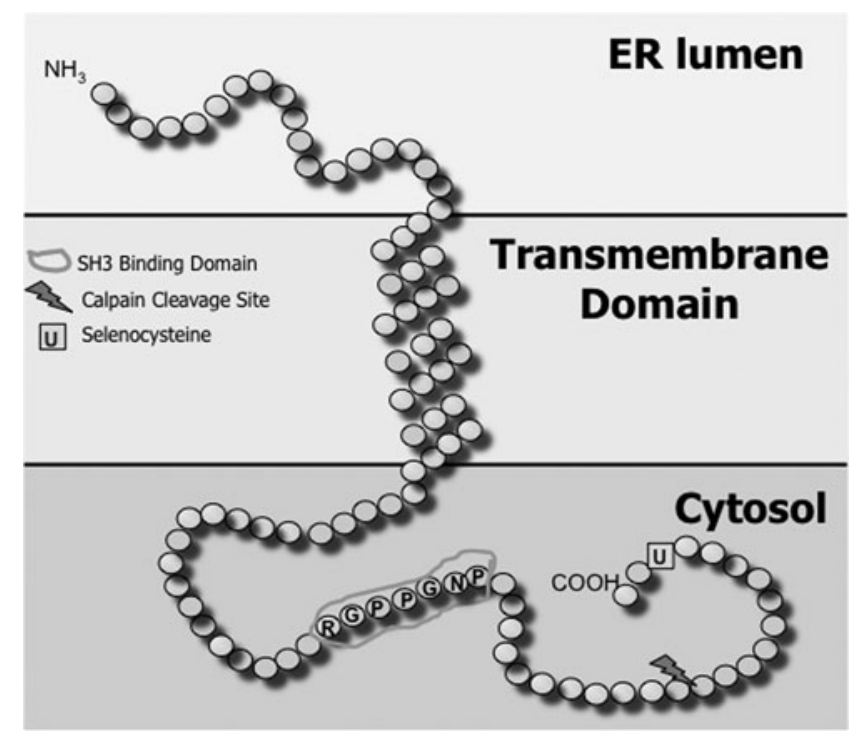

FIG. 1. Predicted structural features of SelK. NCBI aminoacid sequence (NP_064363) was used to predict secondary structure and domains using SOSUI system software, Mitaku group, Nagoya University. Predicted transmembrane domain was confirmed using TMHMM2.0. SelK, Selenoprotein $\mathrm{K}$. 


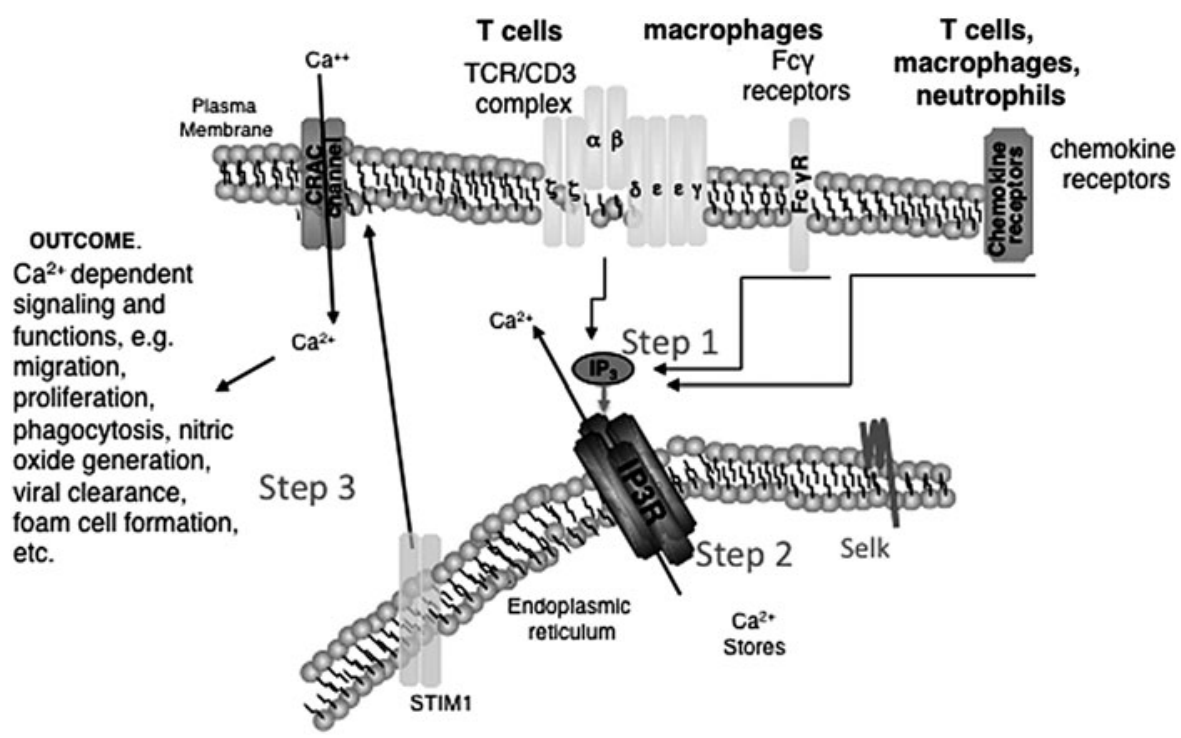

FIG. 2. Diagram of signaling events occurring during receptor-mediated activation of immune cells. Immune receptor engagement by ligands triggers $\mathrm{Ca}^{2+}$ entry from extracellular spaces in three steps. (1) The TCR on T cells, Fc receptors on macrophages, or different chemokine receptors on a variety of immune cells leads to activation of phosphoinositide-specific phospholipase C (PLC $\gamma 1)$. This enzyme catalyzes the degradation of phosphatidylinositol-4,5-bisphosphate to generate inositol-1,4,5-triphosphate (IP3) and diacylglycerol. (2) Binding of IP3 with the IP3 receptor results in release of Ca ${ }^{2+}$ from ER lumen to the cytosol. (3) Loss of $\mathrm{Ca}^{2+}$ from ER causes translocation of STIM1 oligomers toward the plasma membrane where they interact with the pore-forming unit of CRAC channels. CRAC1 (a.k.a. Orai1) activation results in an influx of extracellular $\mathrm{Ca}^{2+}$, which leads to activation of multiple signaling pathways that are indispensable for cellular proliferation, differentiation, and immune cell functions. SelK was found to impact step 2, but not step 1 or 3 . CRAC, Ca ${ }^{2+}$ releaseactivated $\mathrm{Ca}^{2+}$; ER, endoplasmic reticulum; STIM1, stromal interaction molecule 1; TCR, T cell receptor.

capacity to $\mathrm{SH} 3$ containing partner proteins, but lack the Sec required for potential functions of these bound proteins. In this sense, the truncated SelK may act as a dominant negative form of SelK in vivo, and this is a possible scenario that is currently under investigation in our laboratory. Since SelK is the only selenoprotein containing a predicted SH3-binding domain, our investigations into novel functions for SelK have focused on this region and potential interactions with $\mathrm{SH}$ containing proteins. This approach was used to show that the SH3-binding domain in SelK is indeed functional (13) and eventually led to the discovery of SelK as a cofactor in the palmitoylation of target proteins such as the IP3R as described in greater detail next.

\section{SelK-Dependent Palmitoylation of the $\mathrm{Ca}^{2+}$ Channel Protein}

$\mathrm{SelK}^{-1-}$ mice described nearly 5 years ago were found to be healthy and fertile, with no overt phenotype and no signs of ER stress in tissues or cells (43). The most striking effect of SelK deficiency was impaired $\mathrm{Ca}^{2+}$ flux in immune cells, which led us to investigate which step of $\mathrm{Ca}^{2+}$ flux SelK had an impact. A rapid influx of $\mathrm{Ca}^{2+}$ is important during the activation of lymphocytes through the $\mathrm{T}$ - and $\mathrm{B}$-cell receptors, macrophages through $\mathrm{Fc} \gamma$ receptors, and mast cells through Fce receptors, as well as stimulation of various immune cells through chemokine receptors (12). Engagement of immune cell receptors at the plasma membrane leads to the activation of phosphoinositide-specific phospholipase C, which catalyzes the degradation of phosphatidylinositol-4,5biphosphate to generate inositol-1,4,5-triphosphate (IP3) and diacylglycerol (34). IP3 binds to the IP3R, which is a tetrameric $\mathrm{Ca}^{2+}$ channel protein in the ER membrane. The ER is the main $\mathrm{Ca}^{2+}$ store in immune cells (24), and IP3 binding to the IP3R leads to opening of the IP3R channel and release of $\mathrm{Ca}^{2+}$ from ER stores.

The release of $\mathrm{Ca}^{2+}$ from the ER lumen to the cytosol causes structural changes and oligomerization of the ER transmembrane protein, stromal interaction molecule 1 (STIM1) $(15,32)$. These changes allow the cytosolic domain of STIM1 to directly interact with the pore-forming unit, Orai1, of $\mathrm{Ca}^{2+}$ channels on the plasma membrane called $\mathrm{Ca}^{2+}$ release-activated $\mathrm{Ca}^{2+}$ (CRAC) channels. These steps are collectively referred to as store-operated $\mathrm{Ca}^{2+}$ entry (SOCE), and defects in any of the factors involved in SOCE significantly impair immune cell function (11).

SOCE can be divided into three main steps: (1) immune cell receptor engagement inducing the generation of IP3; (2) IP3/IP3R binding leading to loss of $\mathrm{ER} \mathrm{Ca}^{2+}$ stores; and (3) STIM1 inducing CRAC channel opening (Fig. 2). Our published work using thapsigargin to bypass function of the IP3R ruled out a role for SelK in step 3, since this drug induced similar $\mathrm{Ca}^{2+}$ flux in wild-type and $\mathrm{SelK}^{-1-}$ immune cells (43). Subsequently, we found that step 1 was also unaffected as indicated by equivalent levels of both phospholipase $\mathrm{C}$ (PLC) $\gamma$ phosphorylation and IP3 generation in wild-type and $\mathrm{SelK}^{-1-}$ immune cells (13). In contrast, $\mathrm{SelK}^{-1-}$ immune cells were less responsive to exogenously IP3 introduced into the cells compared with wild-type cells, indicating that step 2 (i.e., IP3R function) is the point at which SelK impacts SOCE. Decreased IP3R function was due to decreased levels of the IP3R protein in immune cells and tissues from SelK 
knockout mice compared with wild-type controls. Because we had previously found that SelK was required for palmitoylation and stable expression of another transmembrane protein, CD36, we investigated whether SelK deficiency was affecting palmitoylation of the IP3R protein. Acyl-biotin exchange reactions demonstrated that SelK deficiency induced through genetic excision (i.e., SelK knockout) or through low selenium in cell culture media led to reduced IP3R palmitoylation. Thus, two different transmembrane proteins (IP3R and CD36) rely on SelK for the post-translational addition of the palmitoyl fatty acid moiety, and there are likely more proteins that also depend on this process. This raises the question of how the biochemical steps in S-acylation of target proteins by DHHC6 are influenced by SelK, and different possible mechanisms are described in greater detail next.

\section{The Protein Acyltransferases and the Role of SelK in the Palmitoylation Reaction}

Protein palmitoylation is a post-translational modification in which the 16-carbon fatty acid, palmitic acid, is added to the sulfhydryl group of cysteine residues through a thioester bond (3). The active form of palmitic acid for this reaction is palmitoyl-coenzyme A ( $\mathrm{CoA})$, and CoA is released when the palmitoyl moeity is added via $\mathrm{S}$-acylation to the target cysteine residue on the target protein. This modification can facilitate membrane association of soluble proteins or influence the stable expression of transmembrane proteins $(7,37)$. When initially considering the possible mechanisms by which SelK may regulate protein palmitoylation, the likelihood of SelK directly catalyzing the addition of palmitoyl fatty acid to target proteins seemed quite low given the lack of any conventional acyltransferase catalytic domain within this selenoprotein. It was considered more likely that SelK interacted with one or more of the members of the protein acyltransferase (PAT) family of enzymes that catalyze pro- tein palmitoylation. PATs were only recently identified in yeast (28), and there are 23 mammalian PATs that each contain a common aspartic acid-histidine-histidine-cysteine (DHHC) motif within the catalytic domain (35). SelK is an ER membrane protein, and only two DHHC enzymes are primarily localized to the ER membrane: DHHC4 and DHHC6 (14). In addition, SelK contains an SH3-binding domain and bioinformatic analyses revealed that DHHC6 was the only mammalian PAT containing a predicted $\mathrm{SH} 3$ domain. This suggested that SelK may interact with DHHC6 in the ER membrane to catalyze the palmitoylation of IP3R. Subsequent experiments confirmed that SelK and DHHC6 bind via $\mathrm{SH} 3$-binding domain/SH3 domain interactions and this enzyme/coenzyme complex catalyzes palmitoylation of the IP3R protein, which stabilizes the $\mathrm{Ca}^{2+}$ channel complex in the ER and allows $\mathrm{Ca}^{2+}$ flux to be effectively induced in immune cells (Fig. 3).

After confirming that the IP3R is palmitoylated by DHHC6/SelK, we found that SelK and DHHC6 not only colocalize in the ER membrane, but also their association is dependent on the SH3-binding domain in SelK for interacting with the SH3 domain in DHHC6. Importantly, the SH3binding domain in SelK is not required for its localization to the ER membrane, but it is required for its association with DHHC6. After activation of T cells, the interaction between SelK and DHHC6 is dynamic and this may serve to regulate the turnover or stability of the IP3R or other target proteins. These findings provided a link between IP3R palmitoylation and SelK, although the molecular mechanisms by which SelK's interactions with DHHC mediate protein palmitoylation have not been fully elucidated. It is currently our hypothesis that SelK functions as a coenzyme for DHHC6 in the protein palmitoylation reaction. This is based on the notion that SelK with its bioactive Sec residue binds to DHHC6 and reduces the energy required for the S-acylation of target proteins, thereby increasing the catalytic efficiency of the palmitoylation reaction.
FIG. 3. SelK is a cofactor for palmitoylation of the IP3R catalyzed by DHHC6. Adequate levels of selenium (Se) lead to sufficient expression of SelK, which binds to palmitoyl acyl transferase, DHHC6, to catalyze the palmitoylation of the IP3R. Palmitoyl groups are added via $\mathrm{S}$-acylation to at least three Cys residues in the cytoplasmic region of IP3R, which is required for stable formation of the tetrameric IP3R $\mathrm{Ca}^{2+}$ channel in the ER membrane. IP3R, inositol-1,4,5triphosphate receptor.

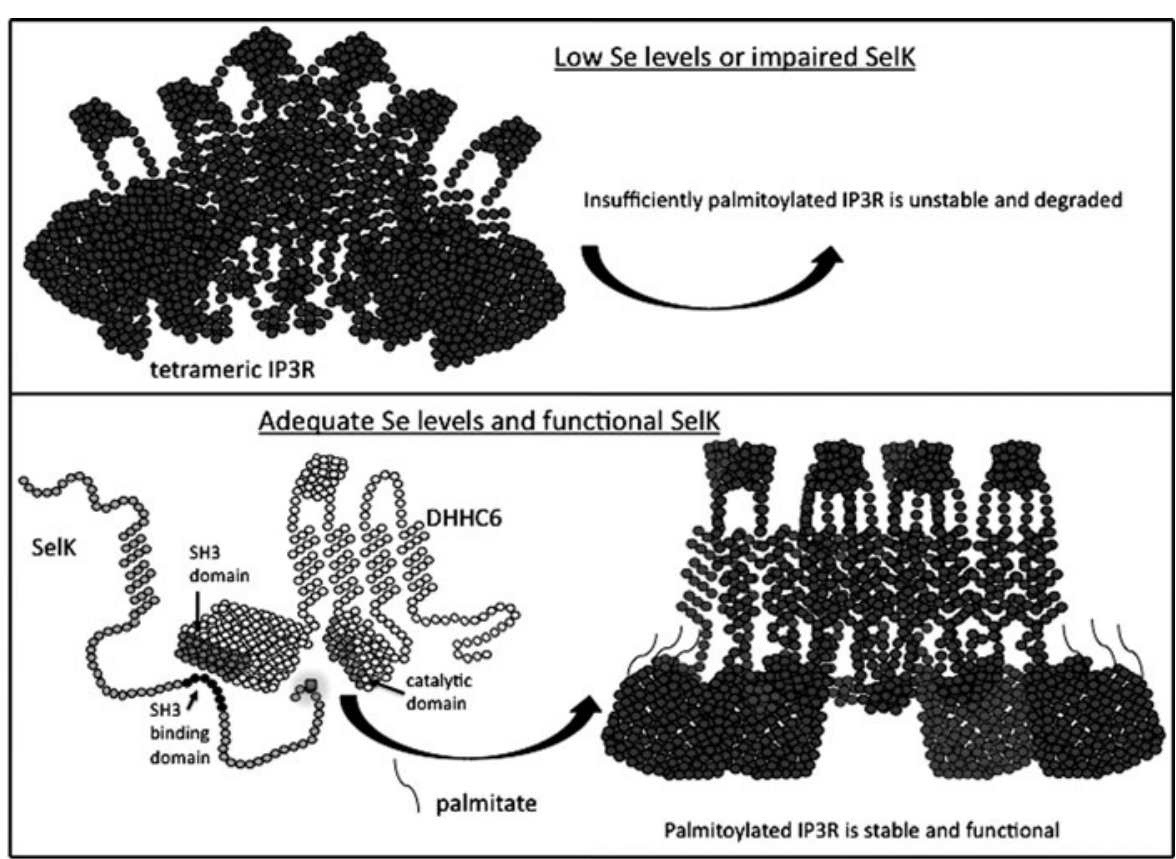




\section{Molecular Mechanisms by Which SelK Functions to Regulate Palmitoylation}

The next few sections describe potential mechanisms of the actions of SelK on protein palmitoylation based on published reports on how DHHC enzymes catalyze S-acylation of target proteins.

\section{Potential mechanism 1: SelK stabilizes the acyl-DHHC6 intermediate in a manner similar to Erf4 (GCP16) and is independent of $\mathrm{Sec}$}

The requirement of a functional coenzyme for efficient PAT activity is not unique to DHHC6. Studies in yeast have shown that the yeast orthologue of DHHC9, Erf2, requires Erf4 (GCP16 in mammals) as a coenzyme $(2,27)$. These studies showed the Erf2/Erf4 protein palmitoylation reaction proceeds via a two-step ping-pong kinetic mechanism. In the first step, a Cys residue within the PAT, presumably the conserved Cys within the DHHC catalytic domain, reacts with palmitoyl-CoA to form a palmitoyl-PAT intermediate. In the second step, the palmitic acid is transferred from the PAT to a Cys residue on the target protein. The first step is rapid, whereas the second is much slower. Thus, after the first step, there is the possibility that the unstable thioester bond between Cys on the PAT and the palmitic acid may hydrolyze before transfer of the palmitic acid to a target protein.

Although there is some evidence that without Erf4 more than one domain of Erf2 is not properly folded leading to activation of more than one ERAD-specific pathway (27), the coenzyme role of Erf4 in the palmitoylation reaction was clearly demonstrated. Specifically, Erf4 was shown to stabilize the thioester bond between Erf2 and palmitic acid as demonstrated by a reduced loss of palmitic acid via hydrolysis and an enhanced transfer to protein substrates. In this manner, SelK may be performing a similar role to Erf4 by stabilizing the thioester bond between palmitic acid and DHHC6 (Fig. 4). If this is the case, one would expect that in the absence of SelK the efficiency of DHHC6-mediated palmitoylation reaction would be reduced due to increased hydrolysis of the intermediate thioester bond between palmitic acid and the Cys within the active site of DHHC6. Given that Erf4 lacks a Sec residue, it is possible that the Sec residue of SelK is not important in this role. In particular, the

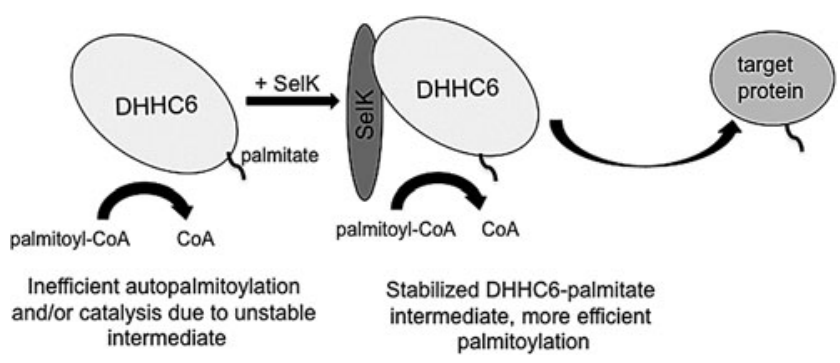

FIG. 4. SelK may stabilize the acyl-DHHC6 intermediate in a manner that may not be dependent on the Sec residue. DHHC6 first interacts with palmitoyl-CoA and becomes autopalmitoylated on a Cys residue. On autopalmitoylation of the DHHC6 enzyme, SelK may stabilize the acyl-DHHC6 intermediate until the target protein can be palmitoylated. CoA, coenzyme A; Sec, selenocysteine. binding of SelK to DHHC6 through the SH3/SH3-binding domains stabilizes the DHHC6-palmitate intermediate independent of the Sec residue in SelK. This would leave the Sec residue free to participate in other functions that SelK may be performing with other protein partners as discussed next.

\section{Potential mechanism 2: A Sec-Cys bond between SelK and DHHC6 is a key modulator of the protein palmitoylation reaction}

The catalytic domain of the PATs, which is a variant of the $\mathrm{C} 2 \mathrm{H} 2$ zinc finger domain, was first recognized in proteins of unknown function in yeast (4). The PAT catalytic domain is cysteine rich and contains a conserved DHHC amino-acid sequence. Indeed, the PAT enzyme family was functionally characterized in yeast before the identification of PAT orthologs in the human genome (28). Further complicating potential investigations into how SelK modulates the palmitoylation reaction is the fact that the identity of the Cys residue within the PAT undergoing autoacylation in the first step of the reaction has yet to be confirmed. While the Cys in the conserved DHHC motif is required for autoacylation, it has been found that three cysteine residues within a CCX7$13 \mathrm{C}(\mathrm{S} / \mathrm{T})$ motif downstream of the DHHC motif can be palmitoylated, leading to the possibility that the conserved Cys is merely required for catalyzing the reaction (45). Given the common theme of Sec-Cys bonding in selenoproteins, it is very possible that the Sec residue of SelK may not only be interacting with the conserved Cys but also with one or several Cys residues in the Cys-rich domain of DHHC6 (Fig. 5). If this is the case, the Sec-Cys bond between SelK and DHHC6 may serve a catalytic function (discussed later) or alternatively could function to modulate the availability of Cys residues within the conserved domain of DHHC6, thereby influencing PAT activity. It is then possible to imagine that the Sec bond between SelK and DHHC6 may have a role in other cellular processes more typically associated with selenoproteins.

\section{Potential mechanism 3: The Sec within SelK undergoes palmitoylation as an additional step in the transfer of palmitic acid from palmitoyl-CoA to the target protein}

As discussed earlier, the PAT reaction is conventionally depicted as a two-step reaction in which the PAT first engages palmitoyl-CoA and undergoes palmitoylation, followed by

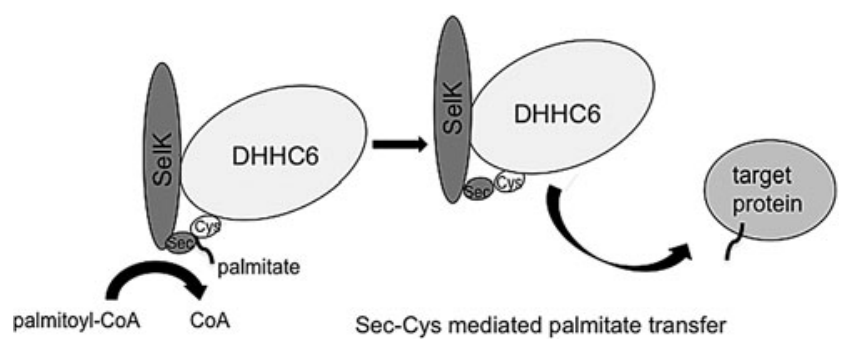

FIG. 5. SelK may form a Sec-Cys bond with DHHC6 to increase efficiency of the palmitoylation reaction. Either the autopalmitoylation step or the transfer of the palmitoyl moiety may occur more effectively through formation of a Sec-Cys bond that acts as a conduit within the active site. 
transfer of the palmitic acid to a target protein. The first step of the reaction is rapid, and the thioester bond between the PAT and palmitic acid is susceptible to hydrolysis before acyl transfer to the target protein. Accordingly, it is possible that the Sec of SelK forms a strong selenoester bond with palmitic acid at some point during the reaction that may limit the amount of palmitic acid lost to hydrolysis (Fig. 6). SelK may undergo Seacylation in the first step of the reaction, followed by acylation of the DHHC6 active site, followed by a third step in which the palmitate is then transferred to a protein target. Another possibility is that DHHC6 is first autoacylated; then, the palmitoyl group is transferred to SelK via Se-acylation in an intermediate step of the reaction, followed by transfer of the palmitate to the target protein. If this is the case, the palmitoylation reaction may occur via formation of several different possible Sec-Cys adducts being formed and broken as palmitate is passed along the course of the reaction from palmitoyl-CoA to the protein target.

\section{Potential mechanism 4: SelK may enhance DHHC6-mediated protein palmitoylation by preventing PAT oligomerization}

Dimer and/or oligomer formation is a common trait of numerous integral membrane proteins. This phenomenon also appears to be common to PATs highlighted by a recent study showing that DHHC2 and DHHC3 tend to form dimers and/or oligomerize in both intact cells and in vitro (30). Bioluminescence resonance energy transfer (BRET) was used to demonstrate that catalytically inactive DHHC proteins displayed a greater propensity to form dimers. Further, when catalytically active DHHC3 preparations were autoacylated in vitro via the addition of palmitoylCoA, a significant decrease in BRET was observed, indicating that the predominantly active state of PATs is the monomeric form. This suggests that enzymatic activity of numerous PATs may be modulated by oligomerization status, which may be one possible mechanism by which SelK modulates DHHC6 activity. The association of SelK with DHHC6 via the SH3 interactions alone may limit and/ or prevent oligomerization of the PAT, thereby keeping it in a more active state (Fig. 7). Sec interactions with one or more Cys residues in the Cys-rich catalytic domain of DHHC6 may further enhance SelK binding and prevent PAT oligomerization. SelK may interact with other PATs

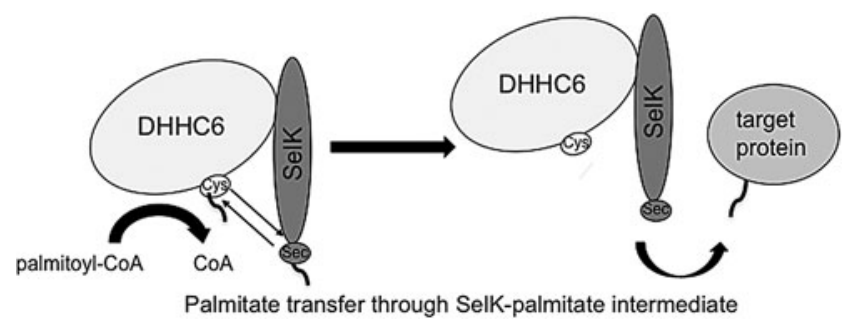

FIG. 6. The Sec within SelK may undergo palmitoylation as an additional step in the transfer of palmitic acid from palmitoyl-CoA to the target protein. The Sec of SelK may be more effective at capturing the palmitate moiety, thereby reducing energy of the first step in the palmitoylation reaction. The palmitoyl group may then be transferred from either the Sec or Cys to the target protein.

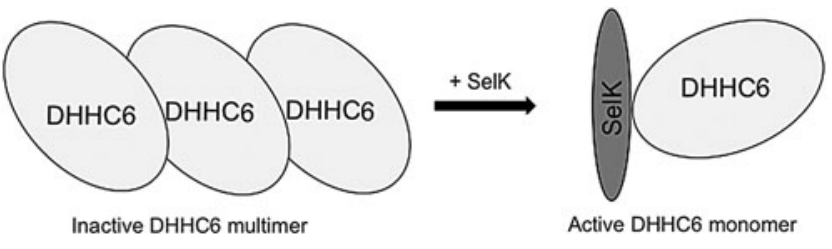

FIG. 7. SelK may enhance DHHC6-mediated protein palmitoylation by preventing PAT oligomerization. Formation of dimers or oligimers has been shown to occur for some PATs, and SelK may prevent DHHC6 oligomerization, thereby increasing the monomeric state of DHHC6 and increasing catalysis of the palmitoylation reaction. PAT, protein acyltransferase.

that do not contain an SH3 domain through Sec-Cys bonds, although how these bonds may be initiated in the absence of docking through the $\mathrm{SH} 3$ and $\mathrm{SH} 3$-binding domain is not clear and is not suggested by our co-immunoprecipitation data (13).

\section{Other Functions of SelK and Potential Relationships with Protein Palmitoylation}

As discussed earlier, purified SelK can dimerize via an intermolecular diselenide bond that exhibits strong reducing potential (22). Purified SelK exhibits some level of peroxidase activity and is able to reduce hydrophobic substrates such as phospholipid hydroperoxides, which is consistent with antioxidant activity displayed by human SelK overexpressed in cells (23). As discussed earlier, the Sec residue of SelK may not participate in the DHHC6-catalyzed protein palmitoylation reaction, which could then leave it free to participate in other reactions such as the peroxidation of lipid peroxides. If this is the case, it is plausible that SelK plays dual roles in the palmitoylation of protein targets. SelK may first direct a quality control role in the protein palmitoylation reaction by reducing lipid hydroperoxides before and/or after they enter the reaction as palmitoyl-CoA substrates. Once the quality control step is complete, SelK may then carry out the second role in increasing the rate and/or fidelity of the DHHC6-catalyzed palmitoylation via one or several of the mechanisms discussed earlier.

The relationship between SelK's role in protein palmitoylation and ERAD might be easier to envision. SelK can protect HepG2 cells against agents that induce ER stress (9). The SelK promoter also contains an ER stress-response element, and the SelK protein interacts with numerous ERAD mediators (e.g., p97 ATPase, Derlins) (38). These studies indicate that SelK plays a multifaceted role in ER homeostasis and protein processing while encompassing much more than its role in protein palmitoylation. These roles may be unrelated, with SelK performing different functions based on the protein complexes in which it participates. This may be supported by data showing that SelK mRNA and protein levels increase during ER stress, and this may provide an abundance of SelK so that both palmitoylation and ERAD functions may be simultaneously carried out. Alternatively, the ER environment may dictate the protein partners to which SelK binds. For instance, at times when ER stress is minimal, SelK may interact predominantly with DHHC6 to perform protein palmitoylation and, when there is an increase in ER 
stress, SelK may bind predominately to ERAD proteins such as p97 ATPase and Derlins to promote protein degradation. In a general manner, SelK may be positioned at the ER membrane for palmitoylation of nascent proteins requiring this post-translational modification for stable conformation or association/insertion into organelle membranes. If palmitoylation does not occur properly, there may be a transfer of unstable proteins to the Derlin chaperones, leading to degradation through the proteosome. While there are currently limited data linking SelK-dependent palmitoylation and ERAD functions, there are some interesting examples in yeast palmitoylation proteins tied to the ERAD machinery. As discussed earlier, Erf2 is a PAT in yeast that interacts with the coenzyme Erf4 to perform protein palmitoylation, and removal of the Erf4 coenzyme leads to hydrolysis of the Erf2palmitoyl intermediate (27). The hydrolyzed Erf2 that increases in the absence of coenzyme Erf4 is degraded through ERAD. This raises the potential scenario in which SelK that binds to DHHC6 that has already hydrolyzed may interact with the Derlin chaperones for proteosomal degradation of those hydrolyzed DHHC6 molecules. This would tie together findings from our studies with those from the Gladyshev laboratory showing interactions of SelK with Derlin chaperones (38).

\section{SelK as a Multifunctional Housekeeping Selenoprotein}

The functions ascribed to human SelK thus far, including palmitoylation, have been mostly housekeeping in nature. Genes encoding housekeeping proteins are often widely expressed in different tissues, which is the case for SelK. While the levels of SelK protein as determined by mouse tissue blots are widely distributed throughout most tissues, much higher levels are found in immune tissues (43). This may reflect a heavy reliance on SelK for basal levels of IP3R palmitoylation in immune cells, which is consistent with a dramatic decrease in IP3R stability in spleens from SelK ${ }^{-1-}$ mice (13). In addition to broad tissue distribution, expression in a wide variety of species (including ancient species) is a characteristic that is common to many housekeeping genes. SelK seems to fit this criterion given that the gene encoding Sec-containing SelK is found in a wide variety of species, including mammals, fish, amphibians, and insects (36).

An interesting point that requires clarification is whether SelK functions as a cofactor for palmitoylating proteins across species other than humans and mice. Interestingly, Drosophila expresses only three Sec-containing proteins, one of which is the orthologue to SelK (dSelK; a.k.a. G-rich protein) (6). Similar to its mammalian counterpart, dSelK contains one Sec residue near the $\mathrm{C}$-terminus and manipulation of dSelK in Drosophila cells alters both IP3R levels and cytoplasmic $\mathrm{Ca}^{2+}$ concentrations (1). Both SelK and $\mathrm{dSelK}$ are rich in prolines and glycines, but the amino-acid sequence of dSelK is lacking the $\mathrm{R} / \mathrm{K}-\mathrm{X}-\mathrm{X}-\mathrm{P}-\mathrm{X}-\mathrm{X}-\mathrm{P}$ motif that defines the canonical SH3-binding domain found in SelK. This may indicate that a mechanism of interaction between dSelK and PATS other than the SH3/SH3-binding domain interaction found in mammals may exist, or that SH3binding domains vary between mammals and insects. The presence of 22 PATs, in the Drosophila genome, including the orthologue to DHHC6, is a strong indicator that protein palmitoylation is important in flies $(21,39)$. In fact, palmitoylation of Hedgehog and other proteins has been demonstrated to play an important role in their functions (5). However, it remains to be determined whether Drosophila orthologues for proteins such as IP3R and CD36 are palmitoylated or whether $\mathrm{dSelK}$ is involved in this process.

Protein palmitoylation is one of the multiple roles that SelK appears to play in human cells, which suggests functional versatility for this relatively small protein. The lens through which functional roles of SelK as well as other selenoproteins are studied may need to be widened. In particular, a comprehensive understanding of the functions of selenoproteins may require probing their interactions with a wide variety of protein partners under different conditions and/or in various cell types. The notion that proteins can carry out multiple functions within cells by binding to different partners or participating in multiprotein complexes is certainly not new and not limited to selenoproteins. The importance of functional versatility is becoming more evident as tools evolve to better resolve protein-protein interactions (42). Publication of the human genome sequence and subsequent annotation and proteomic analyses provided important insights into the versatility of proteins in general. A simple comparison of more recent estimates of $\sim 20,000$ protein-coding genes compared with the prediction of 40,000-100,000 genes before completion of the genome sequence emphasizes the point that multitasking is built into our proteome $(8,10,31)$. In fact, the Human Proteome Project has recently reduced estimates of protein-coding genes to $<20,000$ (20), and the decreasing number further highlights the importance of appreciating the functional versatility of proteins, even small, disordered, and seemingly unremarkable proteins such as SelK.

\section{Conclusions and Loose Ends}

The recent findings that SelK is involved in protein palmitoylation may open a new line of inquiry into potential roles of other selenoproteins as cofactors in enzymatic reactions. While progress has been made in revealing the chemical reactions and factors involved in the palmitoylation of proteins within cells, much remains to be understood about how cofactors such as SelK may influence cell function by regulating the post-translational palmitoylation of different proteins. Some of the many questions that remain are as follows: How many other cellular proteins are palmitoylated in an SelK-dependent manner? Does SelK partner with other DHHC enzymes? What is the role of the Sec residue in the S-acylation reaction?

Studies are ongoing to establish palmitoylation enzyme assays to measure reaction kinetics. We expect that SelK binding to DHHC6 will increase the catalytic efficiency $\left(k_{c a t}\right)$ $\left.K_{m}\right)$ and maximum rate of the system $\left(V_{M A X}\right)$ compared with DHHC6 alone. SelK is also predicted to lower the substrate concentration required for effective DHHC6-driven catalysis to occur $\left(K_{m}\right)$. Moreover, it is of interest to determine whether the $\mathrm{Sec} \rightarrow$ Cys version of SelK will still increase DHHC6 catalytic efficiency compared with DHHC6 alone, but not to levels of Sec-containing SelK. Overall, questions regarding the SelK functions are being answered while more questions emerge, and these muddied waters will gain clarity as mechanistic and biochemical studies progress. 


\section{Acknowledgments}

This research was supported by NIH grant R01AI089999, as well as by P20GM103516, P20RR016453, G12RR003061, and G12MD007601.

\section{Author Disclosure Statement}

No competing financial interests exist.

\section{References}

1. Ben SB, Wang QY, Xia L, Xia JZ, Cui J, Wang J, Yang F, Bai H, Shim MS, Lee BJ, Sun LG, and Chen CL. Selenoprotein $\mathrm{dSelK}$ in Drosophila elevates release of $\mathrm{Ca}^{2+}$ from endoplasmic reticulum by upregulating expression of inositol 1,4,5-trisphosphate receptor. Biochemistry (Mosc) 76: 1030-1036, 2011.

2. Bennett GJ and Sessle BJ. Basic science issues related to improved diagnoses for chronic orofacial pain. Anesth Prog 37: 108-112, 1990.

3. Bijlmakers MJ and Marsh M. The on-off story of protein palmitoylation. Trends Cell Biol 13: 32-42, 2003.

4. Bohm S, Frishman D, and Mewes HW. Variations of the $\mathrm{C} 2 \mathrm{H} 2$ zinc finger motif in the yeast genome and classification of yeast zinc finger proteins. Nucleic Acids Res 25: 2464-2469, 1997.

5. Buglino JA and Resh MD. Palmitoylation of Hedgehog proteins. Vitam Horm 88: 229-252, 2012.

6. Castellano S, Morozova N, Morey M, Berry MJ, Serras F, Corominas M, and Guigo R. In silico identification of novel selenoproteins in the Drosophila melanogaster genome. EMBO Rep 2: 697-702, 2001.

7. Chamberlain LH, Lemonidis K, Sanchez-Perez M, Werno MW, Gorleku OA, and Greaves J. Palmitoylation and the trafficking of peripheral membrane proteins. Biochem Soc Trans 41: 62-66, 2013.

8. Clamp M, Fry B, Kamal M, Xie X, Cuff J, Lin MF, Kellis $\mathrm{M}$, Lindblad-Toh $\mathrm{K}$, and Lander ES. Distinguishing protein-coding and noncoding genes in the human genome. Proc Natl Acad Sci U S A 104: 19428-19433, 2007.

9. Du S, Zhou J, Jia Y, and Huang K. SelK is a novel ER stress-regulated protein and protects HepG2 cells from ER stress agent-induced apoptosis. Arch Biochem Biophys 502: 137-143, 2010.

10. Ezkurdia I, Juan D, Rodriguez JM, Frankish A, Diekhans M, Harrow J, Vazquez J, Valencia A, and Tress ML. Multiple evidence strands suggest that there may be as few as 19000 human protein-coding genes. Hum Mol Genet 23: 5866-5878, 2014.

11. Feske $\mathrm{S}$. Calcium signalling in lymphocyte activation and disease. Nat Rev Immunol 7: 690-702, 2007.

12. Feske $\mathrm{S}$. Immunodeficiency due to defects in store-operated calcium entry. Ann N Y Acad Sci 1238: 74-90, 2011.

13. Fredericks GJ, Hoffmann FW, Rose AH, Osterheld HJ, Hess FM, Mercier F, and Hoffmann PR. Stable expression and function of the inositol 1,4,5-triphosphate receptor requires palmitoylation by a DHHC6/selenoprotein K complex. Proc Natl Acad Sci U S A 111: 16478-16483, 2014.

14. Gorleku OA, Barns AM, Prescott GR, Greaves J, and Chamberlain LH. Endoplasmic reticulum localization of DHHC palmitoyltransferases mediated by lysine-based sorting signals. J Biol Chem 286: 39573-39584, 2011.

15. Hogan PG, Lewis RS, and Rao A. Molecular basis of calcium signaling in lymphocytes: STIM and ORAI. Annu Rev Immunol 28: 491-533, 2010.
16. Huang Z, Hoffmann FW, Fay JD, Hashimoto AC, Chapagain ML, Kaufusi PH, and Hoffmann PR. Stimulation of unprimed macrophages with immune complexes triggers a low output of nitric oxide by calcium-dependent neuronal nitric-oxide synthase. J Biol Chem 287: 4492-4502, 2012.

17. Huang Z, Hoffmann FW, Norton RL, Hashimoto AC, and Hoffmann PR. Selenoprotein K is a novel target of $\mathrm{m}$ calpain, and cleavage is regulated by Toll-like receptorinduced calpastatin in macrophages. J Biol Chem 286: 34830-34838, 2011.

18. Kaneko T, Li L, and Li SS. The SH3 domain - a family of versatile peptide- and protein-recognition module. Front Biosci 13: 4938-4952, 2008.

19. Kryukov GV, Castellano S, Novoselov SV, Lobanov AV, Zehtab O, Guigo R, and Gladyshev VN. Characterization of mammalian selenoproteomes. Science 300: 1439-1443, 2003.

20. Lane L, Bairoch A, Beavis RC, Deutsch EW, Gaudet P, Lundberg E, and Omenn GS. Metrics for the Human Proteome Project 2013-2014 and strategies for finding missing proteins. J Proteome Res 13: 15-20, 2014.

21. Linder ME and Deschenes RJ. New insights into the mechanisms of protein palmitoylation. Biochemistry 42: 4311-4320, 2003.

22. Liu J, Zhang Z, and Rozovsky S. Selenoprotein K form an intermolecular diselenide bond with unusually high redox potential. FEBS Lett 588: 3311-3321, 2014.

23. Lu C, Qiu F, Zhou H, Peng Y, Hao W, Xu J, Yuan J, Wang $\mathrm{S}$, Qiang B, Xu C, and Peng X. Identification and characterization of selenoprotein $\mathrm{K}$ : an antioxidant in cardiomyocytes. FEBS Lett 580: 5189-5197, 2006.

24. Luik RM, Wang B, Prakriya M, Wu MM, and Lewis RS. Oligomerization of STIM1 couples ER calcium depletion to CRAC channel activation. Nature 454: 538-542, 2008.

25. Meiler S, Baumer Y, Huang Z, Hoffmann FW, Fredericks GJ, Rose AH, Norton RL, Hoffmann PR, and Boisvert WA. Selenoprotein $\mathrm{K}$ is required for palmitoylation of CD36 in macrophages: implications in foam cell formation and atherogenesis. J Leukoc Biol 93: 771-780, 2013.

26. Metanis $\mathrm{N}$ and Hilvert D. Natural and synthetic selenoproteins. Curr Opin Chem Biol 22C: 27-34, 2014.

27. Mitchell DA, Hamel LD, Ishizuka K, Mitchell G, Schaefer LM, and Deschenes RJ. The Erf4 subunit of the yeast Ras palmitoyl acyltransferase is required for stability of the Acyl-Erf2 intermediate and palmitoyl transfer to a Ras2 substrate. J Biol Chem 287: 34337-34348, 2012.

28. Mitchell DA, Vasudevan A, Linder ME, and Deschenes RJ. Protein palmitoylation by a family of DHHC protein Sacyltransferases. J Lipid Res 47: 1118-1127, 2006.

29. Morozova N, Forry EP, Shahid E, Zavacki AM, Harney JW, Kraytsberg Y, and Berry MJ. Antioxidant function of a novel selenoprotein in Drosophila melanogaster. Genes Cells 8: 963-971, 2003.

30. Packer CL. Local area networks increase 500\% in 1986. Hospitals 61: 110, 1987.

31. Pennisi E. Human genome. A low number wins the GeneSweep Pool. Science 300: 1484, 2003.

32. Putney JW. Capacitative calcium entry: from concept to molecules. Immunol Rev 231: 10-22, 2009.

33. Reeves MA and Hoffmann PR. The human selenoproteome: recent insights into functions and regulation. Cell Mol Life Sci 66: 2457-2478, 2009.

34. Rhee SG. Regulation of phosphoinositide-specific phospholipase C. Аппи Rev Biochem 70: 281-312, 2001. 
35. Rocks O, Gerauer M, Vartak N, Koch S, Huang ZP, Pechlivanis M, Kuhlmann J, Brunsveld L, Chandra A, Ellinger B, Waldmann $\mathrm{H}$, and Bastiaens PI. The palmitoylation machinery is a spatially organizing system for peripheral membrane proteins. Cell 141: 458-471, 2010.

36. Romagne F, Santesmasses D, White L, Sarangi GK, Mariotti M, Hubler R, Weihmann A, Parra G, Gladyshev VN, Guigo R, and Castellano S. SelenoDB 2.0: annotation of selenoprotein genes in animals and their genetic diversity in humans. Nucleic Acids Res 42: D437-D443, 2014.

37. Salaun C, Greaves J, and Chamberlain LH. The intracellular dynamic of protein palmitoylation. J Cell Biol 191: 1229-1238, 2010.

38. Shchedrina VA, Everley RA, Zhang Y, Gygi SP, Hatfield DL, and Gladyshev VN. Selenoprotein K binds multiprotein complexes and is involved in the regulation of endoplasmic reticulum homeostasis. J Biol Chem 286: 42937-42948, 2011.

39. Steinhauer J, Liu HH, Miller E, and Treisman JE. Trafficking of the EGFR ligand Spitz regulates its signaling activity in polarized tissues. J Cell Sci 126: 4469-4478, 2013.

40. Turanov AA, Shchedrina VA, Everley RA, Lobanov AV, Yim SH, Marino SM, Gygi SP, Hatfield DL, and Gladyshev VN. Selenoprotein $\mathrm{S}$ is involved in maintenance and transport of multiprotein complexes. Biochem J 462: 555-565, 2014.

41. Ursini F, Heim S, Kiess M, Maiorino M, Roveri A, Wissing J, and Flohe L. Dual function of the selenoprotein PHGPX during sperm maturation. Science 285: 1393-1396, 1999.

42. Vakser IA. Protein-protein docking: from interaction to interactome. Biophys J 107: 1785-1793, 2014.

43. Verma S, Hoffmann FW, Kumar M, Huang Z, Roe K, Nguyen-Wu E, Hashimoto AS, and Hoffmann PR. Selenoprotein $\mathrm{K}$ knockout mice exhibit deficient calcium flux in immune cells and impaired immune responses. J Immunol 186: 2127-2137, 2011.

44. Wu MM, Buchanan J, Luik RM, and Lewis RS. $\mathrm{Ca}^{2+}$ store depletion causes STIM1 to accumulate in ER regions closely associated with the plasma membrane. J Cell Biol 174: 803-813, 2006.

45. Yang W, Di Vizio D, Kirchner M, Steen H, and Freeman MR. Proteome scale characterization of human S-acylated proteins in lipid raft-enriched and non-raft membranes. Mol Cell Proteomics 9: 54-70, 2010.

46. Yu H, Chen JK, Feng S, Dalgarno DC, Brauer AW, and Schreiber SL. Structural basis for the binding of prolinerich peptides to SH3 domains. Cell 76: 933-945, 1994.

Address correspondence to:

Dr. Peter R. Hoffmann

Department of Cell and Molecular Biology John A. Burns School of Medicine University of Hawaii 651 Ilalo Street Honolulu, HI 96813

E-mail: peterrh@hawaii.edu

Date of first submission to ARS Central, May 11, 2015; date of acceptance, May 17, 2015.

$\begin{aligned} & \quad \text { Abbreviations Used } \\ & \mathrm{BRET}=\text { bioluminescence resonance energy transfer } \\ & \mathrm{Ca}^{2+}=\text { calcium } \\ & \mathrm{CoA}=\text { coenzyme A } \\ & \mathrm{CRAC}=\mathrm{Ca}^{2+} \text { release-activated } \mathrm{Ca}^{2+} \\ & \mathrm{DHHC}=\text { aspartic acid-histidine-histidine-cysteine } \\ & \mathrm{dSelK}=\text { Drosophila selenoprotein } \mathrm{K} \\ & \mathrm{ER}=\text { endoplasmic reticulum } \\ & \mathrm{ERAD}=\text { ER-associated protein degradation } \\ & \mathrm{IP} 3 \mathrm{R}=\text { inositol-1,4,5-triphosphate receptor } \\ & \mathrm{LDL}=\text { low-density lipoprotein } \\ & \mathrm{PAT}=\text { protein acyltransferase } \\ & \mathrm{Sec}=\text { selenocysteine } \\ & \mathrm{SelK}=\text { Selenoprotein } \mathrm{K} \\ & \mathrm{SH} 3=\text { Src homology } 3 \\ & \mathrm{SOCE}=\text { store-operated Ca } \mathrm{Ca}^{2+} \text { entry } \\ & \mathrm{STIM} 1=\text { stromal interaction molecule } 1 \\ &\end{aligned}$

